\title{
Clinical utility and cost modelling of the phi test to triage referrals into image-based diagnostic services for suspected prostate cancer: the PRIM (Phi to Reflne Mri) study
}

Lois Kim¹, Nicholas Boxall², Anne George ${ }^{3}$, Keith Burling ${ }^{4}$, Pete Acher ${ }^{5}$, Jonathan Aning ${ }^{6}$, Stuart McCracken , Toby Page ${ }^{8}$ and Vincent J. Gnanapragasam ${ }^{2,3,9^{*}}$

\begin{abstract}
Background: The clinical pathway to detect and diagnose prostate cancer has been revolutionised by the use of multiparametric MRI (mpMRI pre-biopsy). mpMRI however remains a resource-intensive test and is highly operator dependent with variable effectiveness with regard to its negative predictive value. Here we tested the use of the phi assay in standard clinical practice to pre-select men at the highest risk of harbouring significant cancer and hence refine the use of mpMRl and biopsies.

Methods: A prospective five-centre study recruited men being investigated through an mpMRI-based prostate cancer diagnostic pathway. Test statistics for PSA, PSA density (PSAd) and phi were assessed for detecting significant cancers using 2 definitions: $\geq$ Grade Group (GG2) and $\geq$ Cambridge Prognostic Groups (CPG) 3. Cost modelling and decision curve analysis (DCA) was simultaneously performed.

Results: A total of 545 men were recruited and studied with a median age, PSA and phi of 66 years, $8.0 \mathrm{ng} / \mathrm{ml}$ and 44 respectively. Overall, $\geq$ GG2 and $\geq$ CPG3 cancer detection rates were 64\% (349/545), 47\% (256/545) and 32\% (174/545) respectively. There was no difference across centres for patient demographics or cancer detection rates. The overall area under the curve (AUC) for predicting $\geq G G 2$ cancers was 0.70 for PSA and 0.82 for phi. AUCs for $\geq$ CPG3 cancers were 0.81 and 0.87 for PSA and phi respectively. AUC values for phi did not differ between centres suggesting reliability of the test in different diagnostic settings. Pre-referral phi cut-offs between 20 and 30 had NPVs of $0.85-0.90$ for $\geq G G 2$ cancers and $0.94-1.0$ for $\geq$ CPG3 cancers. A strategy of mpMRI in all and biopsy only positive lesions reduced unnecessary biopsies by $35 \%$ but missed $9 \%$ of $\geq \mathrm{GG} 2$ and $5 \%$ of $\geq$ CPG3 cancers. Using PH $\geq 30$ to rule out referrals missed $8 \%$ and $5 \%$ of $\geq$ GG2 and $\geq$ CPG3 cancers (and reduced unnecessary biopsies by $40 \%$ ). This was achieved however with $25 \%$ fewer mpMRI. Pathways incorporating PSAd missed fewer cancers but necessitated more unnecessary biopsies. The phi strategy had the lowest mean costs with DCA demonstrating net clinical benefit over a range of thresholds.
\end{abstract}

Conclusion: phi as a triaging test may be an effective way to reduce mpMRI and biopsies without compromising detection of significant prostate cancers.

Keywords: Prostate cancer, Prostate health index (phi), mpMRl, Biopsy, Cambridge prognostic groups

\footnotetext{
* Correspondence: vjg29@cam.ac.uk

${ }^{9}$ Academic Urology Group, Department of Surgery, University of Cambridge, Cambridge, UK

Full list of author information is available at the end of the article
}

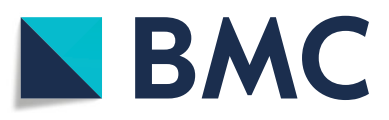

( The Author(s). 2020 Open Access This article is licensed under a Creative Commons Attribution 4.0 International License, which permits use, sharing, adaptation, distribution and reproduction in any medium or format, as long as you give appropriate credit to the original author(s) and the source, provide a link to the Creative Commons licence, and indicate if changes were made. The images or other third party material in this article are included in the article's Creative Commons licence, unless indicated otherwise in a credit line to the material. If material is not included in the article's Creative Commons licence and your intended use is not permitted by statutory regulation or exceeds the permitted use, you will need to obtain permission directly from the copyright holder. To view a copy of this licence, visit http://creativecommons.org/licenses/by/4.0/. The Creative Commons Public Domain Dedication waiver (http://creativecommons.org/publicdomain/zero/1.0/) applies to the data made available in this article, unless otherwise stated in a credit line to the data. 


\section{Background}

Prostate cancer is the most common male cancer in the western world and its incidence is rising. One of the biggest conundrums facing health care systems is how best to detect and diagnose potentially life-limiting disease while not simultaneously over-investigating and finding indolent tumours. The use of multiparametric magnetic resonance imaging (mpMRI) pre-biopsy is now firmly embedded in the prostate cancer diagnostic pathway as a method to try and address this [1-5]. This enables targeting of positive lesions and in the case of negative imaging, avoiding biopsy all together in a proportion of referrals [5]. There remains, however, significant limitation with mpMRI particularly around costs, access, quality control, sustainability and meeting the needs of a growing population of ageing men, only some of whom will harbour lethal disease [6-8]. mpMRI also has significant operator-dependent variability and its negative predictive value is known to vary from study to study depending on scanner type, sequences selected and radiologist experience $[9,10]$. As a result, the European Association of Urology (EAU), amongst other bodies, has called for research into pre-MRI triage tests to refine and improve the use of mpMRI [11]. A few studies have now tested combinations of biomarkers and imaging in controlled trials [12-15]. On-line risk calculators are also available and have recently been updated to include mpMRI data in their predictive algorithms [16]. To date, however, few have considered how biomarker-imaging combinations might work to refine the use of tests and hence cost-effectiveness.

The prostate health index (phi) is one of the most robustly studied prostate cancer biomarkers in the literature and also one of the lowest cost tests available [1719]. It has consistently shown better predictive value compared to PSA in detecting prostate cancer in numerous studies in many countries and has been combined with on-line risk calculators [20]. Previous work in our group was the first to combine phi with mpMRI demonstrating its utility in triaging who needs re-biopsy from an initial negative investigation [21]. Here we have extended that work to test the use of the phi in a multicentre first referral population. Our principle question was if the phi test could reduce mpMRI/biopsy use without compromising detection of prognostically important cancers. Importantly, if this could be done in a routine diagnostic service real-world context without controlling for imaging and biopsy methodology.

\section{Methods}

\section{Patients and data acquisition}

Five UK centres took part with each recruiting consecutive men referred from primary care for elevated prostatespecific antigen (PSA) (January 2018 to June 2019.) mpMRI method and reading and biopsies were carried out according to local protocols in order to ground the study in realworld practices. The only stipulation was image-guided targets + systematic biopsies (cognitive or image fusion). Men were excluded if they had (i) a previous biopsy, (ii) pelvic metalwork interfering with mpMRI quality or no mpMRI and (iii) if no biopsy was done after mpMRI. PSA and phi assay was taken before biopsy and PSA density (PSAd) calculated using mpMRI-defined prostate volumes. Only men with intact information on key data points, PSA $(\mathrm{ng} / \mathrm{mL})$, phi, mpMRI, prostate volume, biopsy performed, histological Grade Group and clinical tumour stage (T-stage) were included in the final analysis. Men with missing data from the original 554 were not included in the analysis leaving a final study cohort of 545 (Additional File Fig. S0). Additional File Table S1 details the imaging and biopsy strategy in each unit. Men with mpMRI-negative lesions had systematic sectoral biopsies only. The study was conducted under ethics REC 03/018.

\section{phi assay}

phi assays were handled according to the manufacturer's recommendations (Beckman Coulter). Blood was taken prior to biopsies and before prostate manipulation. Samples were centrifuged and frozen at $-80{ }^{\circ} \mathrm{C}$ within $3 \mathrm{~h}$ before dispatch to a central laboratory and performed on a Beckman Coulter Access Autoanalyser. Quality Assurance samples were analysed before and after each batch to ensure the validity of the results. All QC results were within Beckman Coulter's target ranges. phi results were not viewed or analysed till after all men had been recruited. There were no adverse events from the extra sampling.

\section{mpMRI}

mpMRI on $1.5 \mathrm{~T}$ or $3 \mathrm{~T}$ systems with multi-channel surface phased array coils were performed including standard anatomical and functional imaging (diffusionweighted and contrast enhanced). Image acquisition and processing was performed in accordance with local standard clinical protocols. In all centres, sequences were evaluated and scored using a Likert scale of cancer probability, based on the Prostate Imaging Reporting and Data ver. 2 (PI-RADS version 2). Prostate volumes were calculated from mpMRI images. Likert 1-2 (M1M2) lesions were considered mpMRI negative for this study and positive lesions graded as M3-M5. Lesion calling was left to local expertise without specification of reader experience.

\section{Statistical analysis and decision modelling}

The primary end-points were two definitions of prognostically important cancers (i.e. cancers that may shorten lifeexpectancy if not found). For this, we used International 
Society of Urological Pathology histological Grade Group 2 or more on biopsy ( $\geq$ GG2) and prognostic group 3 or more using the composite Cambridge Prognostic Group (CPG) prognostic score ( $\geq$ CPG3) we have previously reported and validated [22]. CPG is a five-tiered multi-factor (PSA, Grade and Stage) prognostic model for nonmetastatic prostate cancer shown to have superior discrimination in predicting prostate cancer deaths compared to any other tiered stratification systems [23]. Disease $\geq$ CPG3 is similar to unfavourable intermediate risk and high-risk disease in the AUA, NICE and EAU systems [24]. Areas under the receiver-operating curve (AUC) and diagnostic test statistics for different strategies were compared to PSA alone: phi and PSAd. We adopted a decision modelling approach to bring together information about sensitivity, specificity and costs. A range of potential clinical pathways were modelled and compared Strategy 1: mpMRI and biopsy all, Strategy 2: mpMRI all and biopsy if positive, Strategy 3: mpMRI all and biopsy if PSAd $\geq 0.15$, Strategy 4: mpMRI all and biopsy if PSAd $\geq 0.1$, Strategy 5: phi all and mpMRI and biopsy if phi 25 , Strategy 6: phi all and mpMRI and biopsy if $p h i \geq 30$. The proportions of men with positive test results in the study cohort were used to calculate cancers detected or missed under each pathway. Additional Figure S2 shows the pathway and cohort percentages corresponding to Strategy 6. Costs for assays, scans and biopsies are given in Supplement Table S2; oneway sensitivity analyses were used to explore the impact of assumptions regarding the cost and risk of sepsis following biopsy. Key outcomes relating to correctly identified cancers (true positives), incorrectly identified cancers (false positives), missed cancers (false negatives), numbers of MRI scans, numbers of biopsies and total costs were then calculated for a hypothetical cohort of 1000 referred individuals.

\section{Decision curve analysis}

Decision curve analysis (DCA) was done to estimate the clinical net benefit outcome, which accounts for the perceived value weighting between the harms of biopsy in those without cancer and the harms of not identifying those with cancer [25]. The value weighting represents the decision-makers' beliefs about the benefit to harm ratio between cancer detection and unnecessary biopsy. It can be thought of as the risk of cancer at which there is equipoise about whether to proceed to biopsy (risk threshold, $\mathrm{RT}$ ) and takes values between 0 and 1 . A low risk threshold implies that the perceived harm of biopsy is low compared to the benefits of identifying cancer; conversely, a high-risk threshold occurs when biopsy harm is high compared to the benefits of identifying cancers. The clinical net benefit is then calculated (at a given value of RT) as the number of cancers detected minus the number of unnecessary biopsies weighted by RT. Since the perceived point of biopsy equipoise is uncertain, results are presented over a range of RT values. The preferred detection strategy is then indicated by the one with the highest net benefit, at a given RT. The cost per net cancer detected under each strategy is also presented.

\section{Results}

\section{Cohort description and between centre comparison}

The final study population included 545 men with a median age of 66 years, PSA of $8 \mathrm{ng} / \mathrm{ml}$ and phi of 44 (Table 1). Overall mpMRI results were reported as positive (M3-M5) in 420/545 (77\%) with M4-M5 lesions found in 316/545 (58\%) (Table 1). The median number of cores taken was 16 for systematic biopsies and 2 for targets (if positive on an mpMRI). Overall, prostate cancer was detected in 349/545 men (64\%) and $\geq$ GG2 cancers in 256/545 (47\%). Using the composite CPG score, disease $\geq$ CPG3 was found in 174/545 men (32\%). Additional File Table S1 details the MRI positivity and method of biopsy acquisition for each centre. There were no significant differences between centres in any of these parameters despite no pre-specified standardisation for diagnostic method and reporting [26, 27].

\section{Performance of PSA, PSAd and phi in predicting prostate cancer at biopsy}

For detection of significant cancers defined as $\geq \mathrm{GG} 2$, the AUCs were 0.70, 0.79 and 0.82 for PSA, PSAd and phi respectively (Fig. 1a and Table 2). Both PSAd and phi performed significantly better than PSA alone in predicting $\geq$ GG2 disease $(p<0.001)$ (Table 2$)$. The predictive value of $p h i$ for $\geq$ GG2 was also similar across all 5 centres $(p=0.67)$. The combination of PSA, PSAd with imaging increased the AUC to 0.76 and 0.81 respectively (Table 2). Using $\geq$ CPG3 as an endpoint, the AUCs were $0.81,0.84$ and 0.87 for PSA, PSAd and phi respectively (Additional File Figure S1A and Table 2). However, only phi was significantly better than PSA in predicting $\geq$ CPG3 disease $(p<0.001)$ (Table 2$)$. Amongst mpMRInegative men $(n=125)$, the AUCs for detection of $\geq$ GG2 cancers were $0.64,0.76$ and 0.78 respectively with both PSAd and phi performing better than PSA. Neither PSAd nor phi however performed better than PSA in predicting the presence of $\geq$ CPG3 disease (Fig. 1b, Table 2 and Additional File Figure S1B).

\section{phi thresholds in selecting men for referral to mpMRI and biopsy}

We next tested different threshold of the phi to test its ability to triage men for initial mpMRI and biopsy. Using detection of $\geq$ GG2 as an endpoint, phi cut-offs $\geq 20$ and $\geq 30$ to refer for mpMRI and biopsy had an NPV of 0.85 and 0.90 respectively and missed 1.1 and $7.7 \%$ of tumours (Table 3 ). With $\geq$ CPG3 as a detection target, phi performance showed even better results with NPVs of 
Table 1 Descriptive characteristics of the primary study cohort. MRI positive data is shown as the PI-RADS score of $\geq 3$ or $\geq 4$. Detection rates for cancer are shown for any cancer, and using definitions of $\geq$ Grade Group 2 (GG2 or $\geq$ Cambridge Prognostic Group 3 [CPG3]. Excludes 9 men with missing data not included in the analysis

\begin{tabular}{lc}
\hline Cohort descriptors & $\mathbf{N = 5 4 5}$ \\
\hline Age (median) (inter-quartile range) & $66(60,70)$ years \\
PSA (median) (inter-quartile range) & $8(6,13) \mathrm{ng} / \mathrm{ml}$ \\
PHI (median) (inter-quartile range) & $44(30,69)$ \\
MRI (3-5) & $77 \%$ \\
MRI (4-5) & $58 \%$ \\
Any cancer detection & $64 \%$ \\
$\geq$ GG2 cancer detection & $47 \%$ \\
$\geq$ CPG3 cancer detection & $32 \%$ \\
\hline
\end{tabular}

0.94 and 1.0 respectively and with $0 \%$ and $4.5 \%$ of cancers missed. Of note, using a phi $\geq 35$ threshold (which we reported for re-biopsy men) produced rather poor sensitivity [21] (Table 3). This suggests that useful phi thresholds may be different depending on the detection context. As a comparator, we also analysed the performance of PSAd (noting that this is not usually available before mpMRI). PSAd thresholds of $\geq 0.1, \geq 0.15$ and $\geq 0.2$ missed between 7 and $31 \%$ of $\geq$ GG2 cancers and $3-20 \%$ of $\geq$ CPG3 tumours (Table 3 ).

\section{Impact of using phi as a triaging test into an image- guided diagnostic pathway}

We next compared different pathways to model referrals with and without use of phi. Table 4 summarises the key modelling results for each strategy, based on a hypothetical cohort of 1000 men referred for suspected prostate cancer. The base case model was an mpMRI and biopsy for all which detected every cancer but required more than half of men (53\%) to undergo unnecessary biopsies (i.e. benign histology or $\leq$ GG2) (Table 4). Each alternative option resulted in an overall reduction in biopsy procedures, with the most impactful being a strategy to only biopsy men with positive mpMRI (M3-5) or to only biopsy men with a phi of $\geq 30$ (23\% and $25 \%$ reduction respectively). phi $\geq 30$ also achieved this with a concomitant $25 \%$ reduction in mpMRI use by virtue of being an upfront triage test. We next assessed the impact on rates of detection of significant cancers. Using detection of $\geq$ GG2 as an endpoint, a strategy of mpMRI and biopsy only positive cases reduced unnecessary procedures by $35 \%$ but did miss $9 \%$ of tumours. In contrast, the phi $\geq 30$ option to triage in referrals reduced unnecessary biopsies by $40 \%$ and missed $8 \%$ of $\geq$ GG2 cancers. The phi $\geq 25$ option missed even fewer $\geq$ GG2 cancers (4\%), but only reduced unnecessary biopsies by $25 \%$. Using detection of $\geq$ CPG3 as an endpoint, both strategies of mpMRI and biopsy-positive lesions and using $p h i \geq 30$ led to similarly low missed cancer detection rates (5\%); however, the phi $\geq 30$ route required fewer unnecessary biopsies (31\% vs 35\%). Other pathways using PSAd ( $\geq 0.10$ and 0.15 ) required many more overall biopsies (reductions of only $6-12 \%$ ) and by definition all needed mpMRI (Table 4). These strategies did though miss many fewer $\geq$ GG2 tumours (1-2\%) and no $\geq$ CPG3 disease (Table 4).

\section{Cost modelling and decision curve analysis}

Table 4 includes the mean cost per investigated patient for each of the modelled diagnostic strategies. Compared to a cost of $£ 965 /$ person (mpMRI + biopsy) for all referred men, using a phi $\geq 30$ threshold to restrict investigations reduced costs by $20 \%$ ( $£ 774 /$ person). In contrast, a strategy of mpMRI + biopsy for only scan-positive cases was marginally costlier (£796/person). Strategies that involved using PSAd were also more expensive mainly due to the increased use of both mpMRI and biopsy (mean cost $£ 879$ and $£ 921$ for PSAd $\geq 0.15$ and 0.10 respectively). Sensitivity analyses using higher rate or cost for sepsis following biopsy showed little impact on these comparisons (results not shown). Figure 2 shows the results of the decision curve analysis (incorporating the perceived harms of biopsying those without cancer) in terms of net clinical benefit resulting from different strategies based on $\geq$ GG2 tumour detection. At very low values of the risk threshold, biopsy all is the optimal strategy because these values represent a belief that there is little to no harm associated with an unnecessary biopsy. For risk thresholds $0.2-0.5$ (the estimated range of uncertainty in clinical practice), the net benefit is highest under the $p h i \geq 30$ pathway. Since this is also the cheapest option (Table 4), this appears to be the optimal testing strategy representing a cost/cancer detected of between $£ 2120$ and $£ 5860$ (Additional File Table S3), depending on the risk threshold. However, for risk thresholds $<0.2$ (i.e. where there is uncertainty to biopsy at cancer risk $<20 \%$ ), the clinical net benefit is maximised by using more costly strategies. The optimal decision at these lower-risk thresholds therefore depends on the willingness to pay/cancer detected (Additional File Table S3). Hence for risk thresholds $<0.2$, the cost for the phi $\geq 30$ strategy is around $£ 2000 /$ cancer detected but more cancers (net) could be found using other strategies, at the cost of $£ 4000$ to $£ 8000$ /additional cancer depending on the pathway.

\section{Discussion}

In this paper, we report that use of the phi as a triage test could reduce both imaging and biopsies by a quarter while maintaining diagnostic efficiency using two definitions of clinically significant prostate cancers. We further 


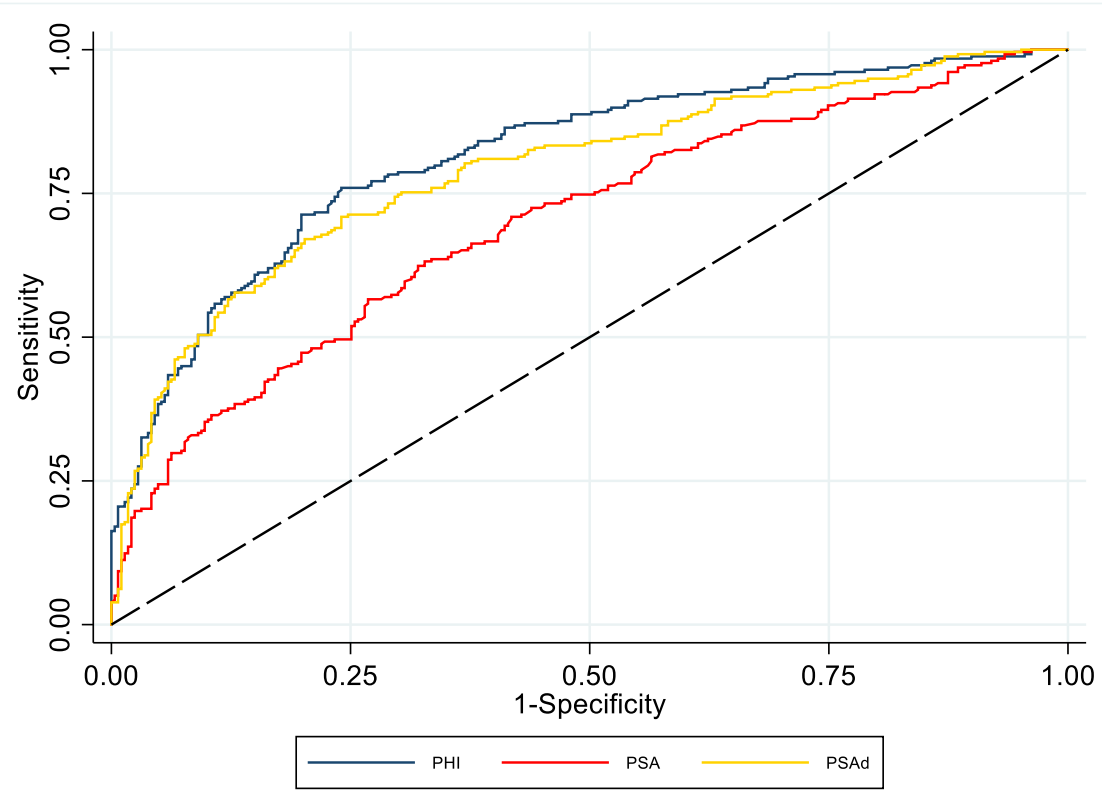

A

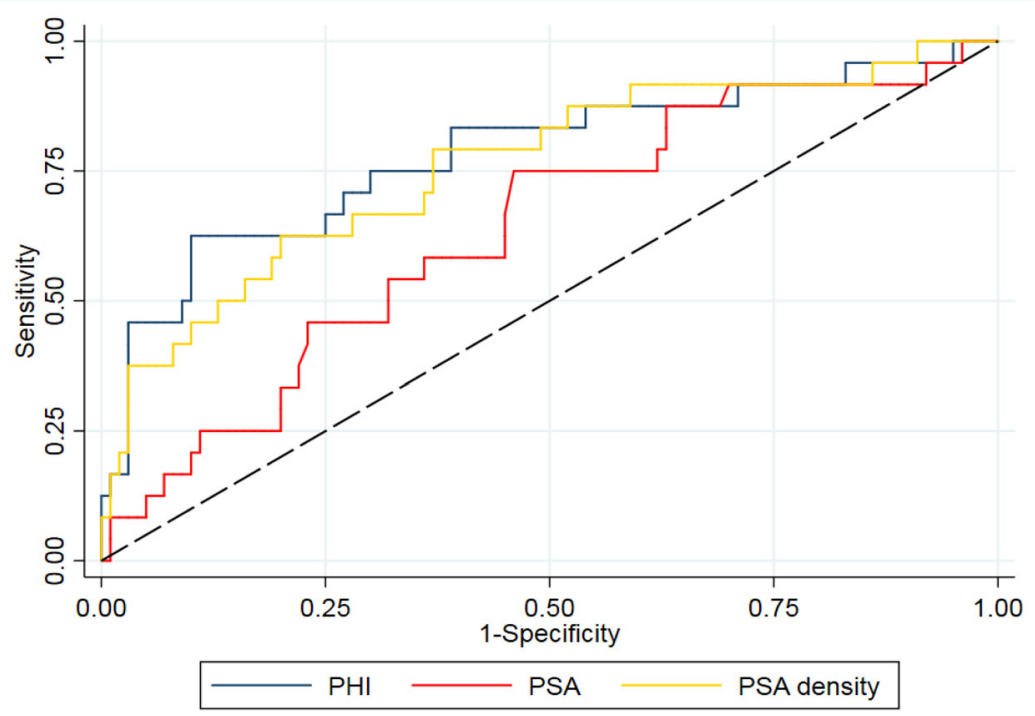

B

Fig. 1 ROC curve illustrating performance of phi, PSA, PSAD and mpMRI in predicting cancer diagnosis of $\geq$ Grade Group 2 (GG2) in the a whole cohort and $\mathbf{b}$ mpMRI-negative men (PI-RADS $\leq 3$ )

demonstrate that introducing the phi is likely to be both the cheapest per referred patient and cheapest per net tumour detected.

The phi is one of many biomarkers reported in the last 10 years that have shown stronger predictive accuracy compared to PSA in detecting prostate cancer [20, 21]. Early studies compared these biomarkers head to head against mpMRI, but inevitably mpMRI proved superior as not only does it improve detection but also helps guide biopsies [28-30]. Subsequent studies have sought to combine biomarkers with mpMRI with all showing consistently better results than biomarkers alone but often in a retrospective setting. One notable prospective study combined the Stockholm3 test with mpMRI and showed the combination was more accurate than each individual test in detecting prostate cancer [14]. These 
Table 2 Descriptive characteristics of the primary study cohort. MRI positive data is shown as the PI-RADS score of $\geq 3$ or $\geq 4$. Detection rates for cancer are shown for any cancer, and using definitions of $\geq$ Grade Group 2 (GG2 or $\geq$ Cambridge Prognostic Group 3 [CPG3]

\begin{tabular}{lcccc}
\hline & $\begin{array}{c}\geq \text { GG2 cancer } \\
\text { detection }\end{array}$ & $\begin{array}{c}\boldsymbol{p} \text { value } \\
\text { (vs. PSA) }\end{array}$ & $\begin{array}{c}\geq \text { CPG3 cancer } \\
\text { detection }\end{array}$ & $\begin{array}{c}\boldsymbol{p} \text { value } \\
\text { (vs.PSA) }\end{array}$ \\
\hline Whole cohort & & & & \\
PSA & $0.70(0.66-0.74)$ & - & $0.81(0.78-0.85)$ & - \\
PSAd & $0.79(0.75-0.83)$ & $<0.001$ & $0.84(0.80-0.87)$ & 0.12 \\
phi & $0.82(0.78-0.85)$ & $<0.001$ & $0.87(0.84-0.90)$ & $<0.001$ \\
MRI & $0.63(0.59-0.66)$ & $<0.001^{*}$ & $0.63(0.60-0.66)$ & $<0.001^{*}$ \\
MRI + PSA & $0.76(0.72-0.80)$ & $<0.001$ & $0.85(0.81-0.88)$ & 0.03 \\
MRI + PSAd & $0.81(0.77-0.84)$ & $<0.001$ & $0.85(0.81-0.88)$ & 0.08 \\
MRI + phi & $0.81(0.78-0.85)$ & $<0.001$ & $0.86(0.83-0.90)$ & 0.02 \\
MRI negative & & & & \\
PSA & $0.64(0.52-0.76)$ & - & $0.86(0.76-0.97)$ & - \\
PSAd & $0.76(0.64-0.87)$ & 0.01 & $0.95(0.91-0.99)$ & 0.08 \\
phi & $0.78(0.66-0.90)$ & 0.01 & $0.89(0.74-1.00)$ & 0.76 \\
\hline
\end{tabular}

studies, however, have generally not considered the incremental cost implications and therefore whether tests can be used sequentially $[11,31]$.

Recent work improving risk calculator performance using biomarkers and/or mpMRI have tended to use prostate biopsy as the end point $[32,33]$. One exception is the work by Mannaerts et al. who retrospectively applied the Rotterdam Prostate Cancer risk calculator in 200 men and proposed that the calculator could have reduced mpMRI by 37\% [34]. An updated calculator incorporating mpMRI was developed but in a recent prospective study did not improve prediction in biopsy naïve men but did do so for a re-biopsy population [35]. This suggests that simply modifying existing calculators with new parameters may need careful re-evaluation to test applicability. Particularly, if once "free" calculators start to incorporate costly biomarkers and imaging data points. In this regard, an advantage of our current study is its prospective application in a real-world setting and simultaneous cost analysis.

Our cost modelling suggests that a phi-based triage pathway may be less costly than other existing strategies. This is particularly relevant as mpMRI is now a mandatory prerequisite step before prostate biopsy and hence an essential diagnostic cost [5]. Bi-parametric instead of multiparametric MRI may be cheaper but their comparative effectiveness remains debated [36, 37]. The cost of biomarkers also remains controversial with different studies showing varying results [38-40]. It is however notable that the phi test as costed by the manufacturer appears to be the most affordable amongst available biomarkers [19]. In this study, we found that it was both the cheapest per referred patient and the cheapest per tumour detected. Although other strategies (mpMRI and biopsy all or using PSAd) detected more prostate cancers, it came at a significantly higher cost as a result of having to undertake many more procedures.

This paper has many strengths. Key is the multicentre design in five different image-guided standard of care prostate diagnostic pathways. These are also limitations as there was no central quality assurance of biopsy method, histology and imaging. We also cannot account for decisions to not do biopsy if an mpMRI was negative as practice differed amongst sites and was evolving. Despite this, we were reassured to see that cancer detection rates and phi test performance were similar across centres. Detection rates were also comparable if not higher than many other published series which have used much more stringent trial parameters [41-43]. Our cost modelling was

Table 3 Diagnostic test statistics of the study cohort showing the accuracy and missed cancer rates for each phi threshold as a triage test pre-MRI and biopsy. PSAd thresholds are shown as a comparator though this metric is not usually available before an mpMRI. Detection rates for cancer are shown using definitions of $\geq$ Grade Group 2 (GG2) or $\geq$ Cambridge Prognostic Group 3 [CPG3]. ( ${ }^{*}$ percentage out of 258 cancers detected, ** percentage out of 176 cancers detected)

\begin{tabular}{|c|c|c|c|c|c|c|c|c|c|}
\hline & \multicolumn{5}{|c|}{$\geq$ GG2 cancer detection } & \multicolumn{4}{|c|}{$\geq$ CPG3 cancer detection } \\
\hline & Threshold & Sensitivity & Specificity & NPV & $\begin{array}{c}\% \text { cancers } \\
\text { missed }^{*}\end{array}$ & Sensitivity & Specificity & NPV & $\begin{array}{l}\% \text { cancers } \\
\text { missed }^{* *}\end{array}$ \\
\hline \multirow[t]{4}{*}{ phi } & 20 & 0.99 & 0.10 & 0.90 & 1.1 & 1.00 & 0.08 & 1.00 & 0 \\
\hline & 25 & 0.96 & 0.25 & 0.87 & 4.2 & 0.99 & 0.22 & 0.99 & 0.05 \\
\hline & 30 & 0.92 & 0.40 & 0.85 & 7.7 & 0.95 & 0.35 & 0.94 & 1.0 \\
\hline & 35 & 0.87 & 0.55 & 0.83 & 12.8 & 0.93 & 0.49 & 0.93 & 7.3 \\
\hline \multirow[t]{3}{*}{ PSAd } & 0.10 & 0.93 & 0.31 & 0.82 & 7.3 & 0.97 & 0.28 & 0.95 & 2.8 \\
\hline & 0.15 & 0.81 & 0.57 & 0.77 & 18.6 & 0.90 & 0.53 & 0.92 & 9.6 \\
\hline & 0.20 & 0.69 & 0.77 & 0.73 & 31.3 & 0.80 & 0.72 & 0.88 & 20.0 \\
\hline
\end{tabular}


Table 4 Modelling results for a hypothetical cohort of 1000 patients referred for elevated PSA using different strategies for cancer detection. In brackets are projected reductions compared to the base model (* compared to MRI + biopsy all)

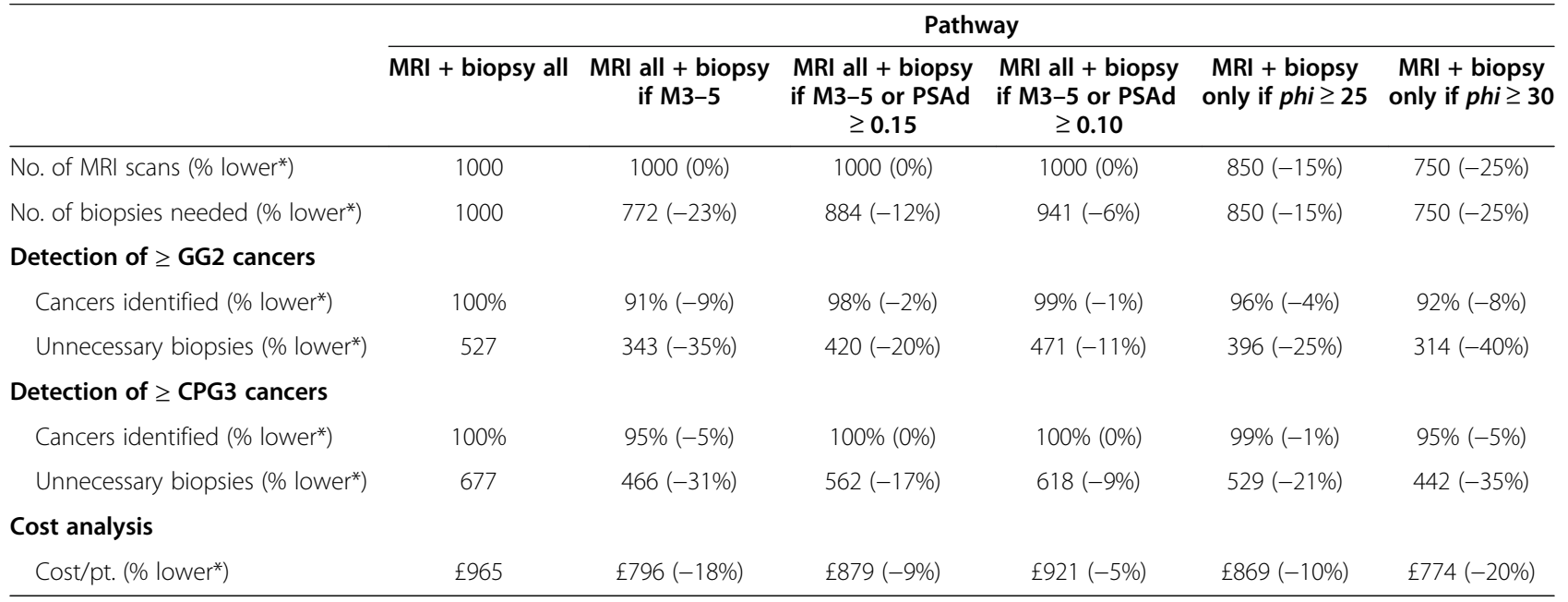

based on UK tariffs and extrapolation to other settings is dependent on individual tariffs in other countries. We note however that mpMRI-phi cost differentials are much greater in Europe and the USA. We did not compare performance of the phi with other biomarkers as these were not available to us. The Stockholm3 study showed very comparable results with a $10 \%$ risk threshold reducing mpMRI and biopsies by $40 \%$ and missing $8 \%$ of cancers [14]. A cost analysis was not published.

\section{Conclusion}

We present here a first study reporting use of the phi test as a way of refining and reducing both mpMRI and biopsies in investigating suspected prostate cancer. Sequential use of the phi and then mpMRI \pm biopsy may therefore be an efficient and effective way of identifying those men who will benefit most from investigation hence reducing cost and resource use in a rapidly growing disease demographic.

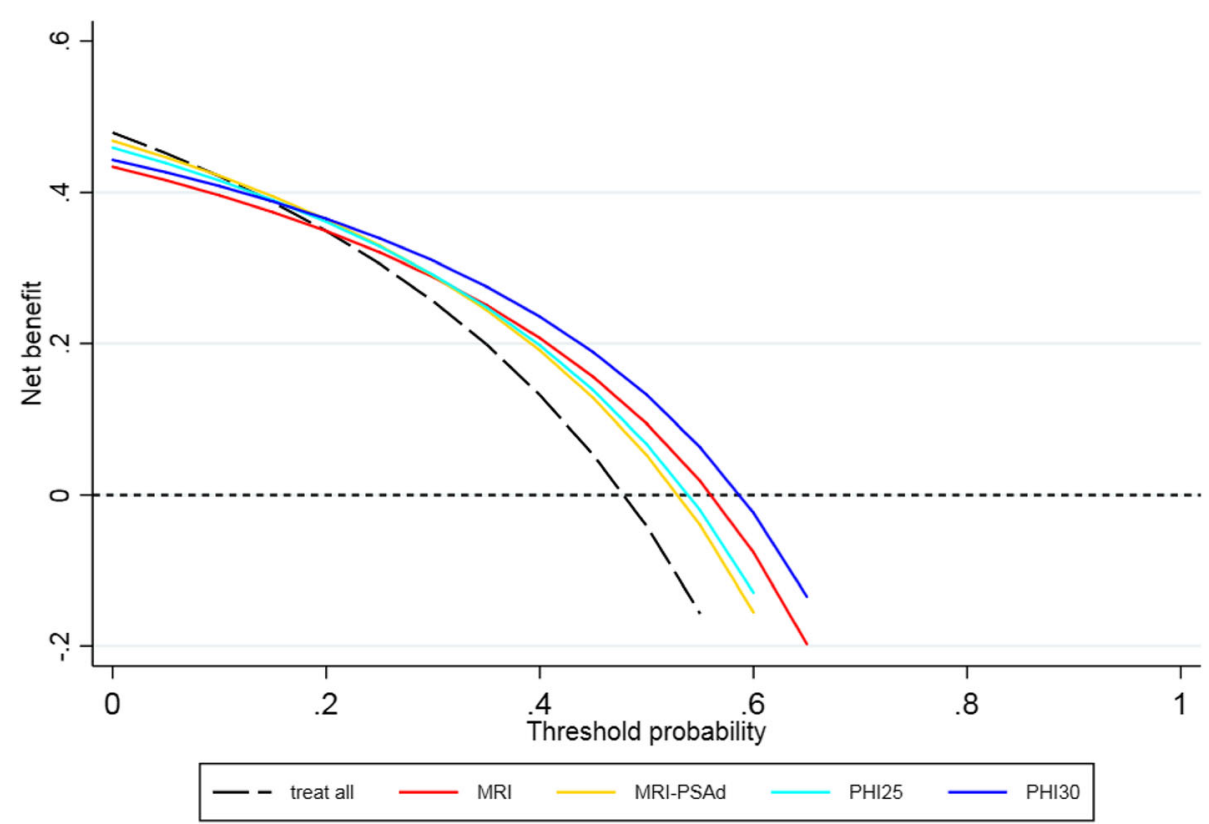

Fig. 2 Decision curve analysis comparing the number of net benefits for detection of significant cancers for a range of risk threshold values and using different approaches (MRI-PSAd using a PSAd threshold of $\geq 0.15$ ). MRI - magnetic resonance imaging, PSAd - PSA density, phi - Prostate Health Index 


\section{Supplementary information}

Supplementary information accompanies this paper at (https://doi.org/10. 1186/s12916-020-01548-3).

\begin{abstract}
Additional file 1: Figure So. Flow chart of recruitment and tests and final numbers analysed. Figure S1. ROC curve illustrating performance of PHI, PSA, PSAD and mpMRI in predicting cancer diagnosis of Cambridge Prognostic Group 3 [CPG3] in A. Whole cohort and B. mpMRI negative men (PI-RADS $\leq 3)$. Figure S2. Decision tree pathway and percentages based on phi>30 pathway. Table S1. Profile of imaging and diagnostic method at each of the centres in the study. Table S2. Model parameters: base case values. Costs ascribed for each event. Table S3. Costs per net cancer detected, by risk threshold. It is assumed that at risk thresholds $>0.5$ (risk of cancer $>50 \%$ ), there would be clinical consensus that the perceived harms of biopsy are outweighed by the benefits of detecting and treating any cancer).
\end{abstract}

\section{Acknowledgements}

We are grateful for the assistance of Dr. Tristan Barret, Dr. Anne Warren, Sr Lorraine Starling (Cambridge), Dr. Sid Liyanage, Dr. Konrad Wolfe (Southend), Sr Connie Shiridzinomwa, Sr Carol Brain (Bristol), Sr Susan Asterling, Sr Deborah Kemp, Dr. Ben Hall (South Tyneside and Sunderland), and the Urology Research team Freeman Hospital (Newcastle). We are also grateful to all clinicians and nurse specialists in the trial sites for access to patients and help in facilitating recruitment.

\section{Authors' contributions}

VJG contributed to the study inception, design, data collation and analysis and manuscript writing. LK contributed to the study analysis and manuscript writing. PA, JA, SM and TB contributed to the data collation and manuscript writing. $\mathrm{KMB}$ contributed to the sample analysis, data collation and manuscript writing AG contributed to the study co-ordination and data collation. NB contributed to the manuscript writing. All authors read and approved the final manuscript.

\section{Funding}

This work was supported by an Addenbrookes Charitable Trust Innovation Grant and an unrestricted education grant for reagents from Beckman Coulter. Neither body had any influence or role in study design, execution, analysis, manuscript drafting, or reporting. Infrastructure funding is acknowledged from CRUK Cambridge Cancer Centre and the Cambridge Biomedical Campus.

\section{Availability of data and materials}

All data is available by application to the study authors.

\section{Ethics approval and consent to participate}

The study was conducted under ethics REC 03/018. All participants consented to anonymised blood samples and clinical data being used for this study.

\section{Competing interests}

VJG has previously received a speaker honorarium from Beckman Coulter on biomarkers in prostate cancer prior to the conception of this study. The other authors declare that they have no competing interests.

\footnotetext{
Author details

${ }^{1}$ Department of Public Health and Primary Care, University of Cambridge, Cambridge, UK. ${ }^{2}$ Department of Urology, Cambridge University Hospitals Trust, Cambridge, UK. ${ }^{3}$ Urological Malignancies Programme CRUK \& Cambridge Urology Translational Research and Clinical Trials Office, University of Cambridge Box 193, Cambridge Biomedical Campus Cambridge CB20QQ, Cambridge, UK. ${ }^{4} \mathrm{NIHR}$ Cambridge Biomedical Research Centre, Core Biochemical Assay Laboratory, University of Cambridge, Cambridge, UK. ${ }^{5}$ Department of Urology, Southend Hospital, Essex, UK. ${ }^{6}$ Department of Urology, North Bristol NHS Trust, Bristol, UK. ${ }^{7}$ Department of Urology, South Tyneside and Sunderland NHS Trust, Sunderland, UK. ${ }^{8}$ Department of Urology, Newcastle Hospitals NHS Trust, Newcastle upon Tyne, UK. ${ }^{9}$ Academic Urology Group, Department of Surgery, University of Cambridge, Cambridge, UK.
}

Received: 14 December 2019 Accepted: 3 March 2020

Published online: 17 April 2020

\section{References}

1. Davies C, Castle JT, Stalbow K, Haslam PJ. Prostate mpMRI in the UK: the state of the nation. Clin Radiol. 2019;74(11):894.e11-8. https://doi.org/10. 1016/j.crad.2019.09.129 Review.

2. Brizmohun Appayya M, Adshead J, Ahmed HU, Allen C, Bainbridge A, Barrett T, Giganti F, Graham J, Haslam P, Johnston EW, Kastner C, Kirkham APS, Lipton A, McNeill A, Moniz L, Moore CM, Nabi G, Padhani AR, Parker C, Patel A, Pursey J, Richenberg J, Staffurth J, van der Meulen J, Walls D, Punwani S. National implementation of multi-parametric magnetic resonance imaging for prostate cancer detection - recommendations from a UK consensus meeting. BJU Int. 2018;122(1):13-25.

3. Rouviere O, Moldovan PC. The current role of prostate multiparametric magnetic resonance imaging. Asian J Urol. 2019;6(2):137-45.

4. https://uroweb.org/guideline/prostate-cancer/. Accessed 11112019.

5. Dasgupta P, Davis J, Hughes S. NICE guidelines on prostate cancer 2019. BJU Int. 2019;124(1):1.

6. Smith-Palmer J, Takizawa C, Valentine W. Literature review of the burden of prostate cancer in Germany, France, the United Kingdom and Canada. BMC Urol. 2019;19(1):19.

7. Rawla P. Epidemiology of prostate cancer. World J Oncol. 2019;10(2):63-89. https://doi.org/10.14740/wjon1191.

8. Rider JR, Sandin F, Andrén O, Wiklund P, Hugosson J, Stattin P. Long-term outcomes among noncuratively treated men according to prostate cancer risk category in a nationwide, population-based study. Eur Urol. 2013;63(1): 88-96.

9. Kohestani K, Wallström J, Dehlfors N, Sponga OM, Månsson M, Josefsson A, Carlsson S, Hellström M, Hugosson J. Performance and inter-observer variability of prostate MRI (PI-RADS version 2) outside high-volume centres. Scand J Urol. 2019:29:1-8.

10. Pickersgill NA, Vetter JM, Andriole GL, Shetty AS, Fowler KJ, Mintz AJ, Siegel CL, Kim EH. Accuracy and variability of prostate multiparametric magnetic resonance imaging interpretation using the prostate imaging reporting and data system: a blinded comparison of radiologists. Eur Urol Focus. 2020;6(2):267-72.

11. Moldovan PC, Van den Broeck T, Sylvester R, Marconi L, Bellmunt J, van denBergh RCN, Bolla M, Briers E, Cumberbatch MG, Fossati N, Gross T, Henry AM, Joniau S, van der Kwast TH, Matveev VB, van der Poel HG, De Santis M, Schoots IG, Wiegel T, Yuan CY, Cornford P, Mottet N, Lam TB, Rouvière O. What is the negative predictive value of multiparametric magnetic resonance imaging in excluding prostate cancer at biopsy? A systematic review and meta-analysis from the European Association of Urology Prostate Cancer Guidelines Panel. Eur Urol. 2017;72(2):250-66.

12. Busetto GM, De Berardinis E, Sciarra A, Panebianco V, Giovannone R, Rosato S, D'Errigo P, Di Silverio F, Gentile V, Salciccia S. Prostate cancer gene 3 and multiparametric magnetic resonance can reduce unnecessary biopsies: decision curve analysis to evaluate predictive models. Urology. 2013;82(6):1355-60.

13. Marzouk K, Ehdaie B, Vertosick E, Zappala S, Vickers A. Developing an effective strategy to improve the detection of significant prostate cancer by combining the 4Kscore and multiparametric MRI. Urol Oncol. 2019;37(10):672-7.

14. Grönberg $H$, Eklund $M$, Picker W, Aly M, Jäderling F, Adolfsson J, Landquist M, Haug ES, Ström P, Carlsson S, Nordström T. Prostate cancer diagnostics using a combination of the Stockholm3 blood test and multiparametric magnetic resonance imaging. Eur Urol. 2018;74(6):722-8.

15. Hendriks RJ, van der Leest MMG, Dijkstra S, Barentsz JO, Van Criekinge W, Hulsbergen-van de Kaa CA, Schalken JA, Mulders PFA, van Oort IM. A urinary biomarker-based risk score correlates with multiparametric MRI for prostate cancer detection. Prostate. 2017;77(14):1401-7.

16. Schoots IG, Padhani AR. Personalizing prostate cancer diagnosis with multivariate risk prediction tools: how should prostate MRI be incorporated? World J Urol. 2019. https://doi.org/10.1007/s00345-019-02899-0.

17. Kohaar I, Petrovics G, Srivastava S. A rich array of prostate cancer molecular biomarkers: opportunities and challenges. Int J Mol Sci. 2019;20(8):1813. https://doi.org/10.3390/ijms20081813.

18. Lamy PJ, Allory Y, Gauchez AS, Asselain B, Beuzeboc P, de Cremoux P, Fontugne J, Georges A, Hennequin C, Lehmann-Che J, Massard C, Millet I, Murez T, Schlageter MH, Rouvière O, Kassab-Chahmi D, Rozet F, Descotes JL, Rébillard X. Prognostic biomarkers used for localised prostate cancer management: a systematic review. Eur Urol Focus. 2018;4(6):790-803. 
19. Mathieu R, Castelli C, Fardoun T, Peyronnet B, Shariat SF, Bensalah K Vincendeau S. Cost analysis of prostate cancer detection including the prostate health index (phi). World J Urol. 2019;37(3):481-7.

20. Wang W, Wang M, Wang L, Adams TS, Tian Y, Xu J. Diagnostic ability of \%p2PSA and prostate health index for aggressive prostate cancer: a metaanalysis. Sci Rep. 2014;4:5012. https://doi.org/10.1038/srep05012.

21. Gnanapragasam VJ, Burling K, George A, Stearn S, Warren A, Barrett T, Koo B, Gallagher FA, Doble A, Kastner C, Parker RA. The prostate health index adds predictive value to multi-parametric MRI in detecting significant prostate cancers in a repeat biopsy population. Sci Rep. 2016;6:35364.

22. Gnanapragasam VJ, Bratt $O$, Muir K, Lee LS, Huang HH, Stattin P, Lophatananon A. The Cambridge Prognostic Groups for improved prediction of disease mortality at diagnosis in primary non-metastatic prostate cancer: a validation study. BMC Med. 2018;16(1):31

23. Zelic R, Garmo H, Zugna D, Stattin P, Richiardi L, Akre O, Pettersson A. Predicting prostate cancer death with different pretreatment risk stratification tools: a head-to-head comparison in a nationwide cohort study. Eur Urol. 2019.

24. http://www.auanet.org/guidelines/clinically-localized-prostate-cancer-newaua/astro/suo-guideline-2017. Accessed 121119.

25. Vickers AJ, Elkin EB. Decision curve analysis: a novel method for evaluating prediction models. Med Decis Mak. 2006;26(6):565-74.

26. Batura D, Gopal RG. The national burden of infections after prostate biopsy in England and Wales: a wake-up call for better prevention--authors' response. J Antimicrob Chemother. 2013;68(10):2419-20.

27. Hex N, Retzler J, Bartlett C, Arber M. The cost of sepsis care in the UK: York Health Economics Consortium; 2017. http://allcatsrgrey.org.uk/wp/wpfb-file/ yhec-sepsis-report-17-02-17-final-pdf. Last accessed 13 Dec 2018.

28. Porpiglia F, Russo F, Manfredi M, Mele F, Fiori C, Bollito E, Papotti M, Molineris I, Passera R, Regge D. The roles of multiparametric magnetic resonance imaging, PCA3 and prostate health index-which is the best predictor of prostate cancer after a negative biopsy? J Urol. 2014;192(1):60-6.

29. Sciarra A, Panebianco V, Cattarino S, Busetto GM, De Berardinis E, Ciccariello M, Gentile V, Salciccia S. Multiparametric magnetic resonance imaging of the prostate can improve the predictive value of the urinary prostate cancer antigen 3 test in patients with elevated prostate-specific antigen levels and a previous negative biopsy. BJU Int. 2012;110(11):1661-5.

30. Osses DF, Roobol MJ, Schoots IG. Prediction medicine: biomarkers, risk calculators and magnetic resonance imaging as risk stratification tools in prostate cancer diagnosis. Int J Mol Sci. 2019;20(7):E1637.

31. Ling DI, Lynd LD, Harrison M, Anis AH, Bansback N. Early cost-effectiveness modeling for better decisions in public research investment of personalized medicine technologies. J Comp Eff Res. 2019;8(1):7-19.

32. Verbeek JFM, Bangma CH, Kweldam CF, van der Kwast TH, Kümmerlin IP, van Leenders GJLH, Roobol MJ. Reducing unnecessary biopsies while detecting clinically significant prostate cancer including cribriform growth with the ERSPC Rotterdam risk calculator and 4Kscore. Urol Oncol. 2019;37(2):138-44.

33. Loeb S, Shin SS, Broyles DL, Wei JT, Sanda M, Klee G, Partin AW, Sokoll L, Chan DW, Bangma CH, van Schaik RHN, Slawin KM, Marks LS, Catalona WJ. Prostate Health Index improves multivariable risk prediction of aggressive prostate cancer. BJU Int. 2017;120(1):61-8.

34. Mannaerts CK, Gayet M, Verbeek JF, Engelbrecht MRW, Savci-Heijink CD, Jager GJ, Gielens MPM, van der Linden H, Beerlage HP, de Reijke TM, Wijkstra H, Roobol MJ. Prostate cancer risk assessment in biopsy-naive patients: the Rotterdam prostate cancer risk calculator in multiparametric magnetic resonance imaging-transrectal ultrasound (TRUS) fusion biopsy and systematic TRUS biopsy. Eur Urol Oncol. 2018;1(2):109-17.

35. Alberts AR, Roobol MJ, Verbeek JFM, Schoots IG, Chiu PK, Osses DF, Tijsterman JD, Beerlage HP, Mannaerts CK, Schimmöller L, Albers P, Arsov C. Prediction of high-grade prostate cancer following multiparametric magnetic resonance imaging: improving the Rotterdam European randomized study of screening for prostate cancer risk calculators. Eur Urol. 2019;75(2):310-8.

36. Kang Z, Min X, Weinreb J, Li Q, Feng Z, Wang L. Abbreviated biparametric versus standard multiparametric MRI for diagnosis of prostate cancer: a systematic review and meta-analysis. AJR Am J Roentgenol. 2019;212(2):357-65.

37. Walz J. Let's keep it at one step at a time: why biparametric magnetic resonance imaging is not the priority today. Eur Urol. 2019;76(5):582-3.

38. Heijnsdijk EA, Denham D, de Koning HJ. The cost-effectiveness of prostate cancer detection with the use of prostate health index. Value Health. 2016; 19(2):153-7.
39. Nichol MB, Wu J, Huang J, Denham D, Frencher SK, Jacobsen SJ. Costeffectiveness of prostate health index for prostate cancer detection. BJU Int. 2012;110(3):353-62.

40. Sathianathen NJ, Kuntz KM, Alarid-Escudero F, Lawrentschuk NL, Bolton DM Murphy DG, Weight CJ, Konety BR. Incorporating biomarkers into the primary prostate biopsy setting: a cost-effectiveness analysis. J Urol. 2018; 200(6):1215-20

41. Rouvière $O$, Puech $P$, Renard-Penna R, Claudon M, Roy C, Mège-Lechevallier $F$, Decaussin-Petrucci M, Dubreuil-Chambardel M, Magaud L, Remontet L, Ruffion A, Colombel M, Crouzet S, Schott AM, Lemaitre L, Rabilloud M, Grenier N, MRI-FIRST Investigators. Use of prostate systematic and targeted biopsy on the basis of multiparametric MRI in biopsy-naive patients (MRI-FIRST): a prospective, multicentre, paired diagnostic study. Lancet Oncol. 2019;20(1):100-9.

42. van der Leest $M$, Cornel E, Israël B, Hendriks R, Padhani AR, Hoogenboom M, Zamecnik P, Bakker D, Setiasti AY, Veltman J, van den Hout $H$, van der Lelij H, van Oort I, Klaver S, Debruyne F, Sedelaar M, Hannink G, Rovers M, Hulsbergen-van de Kaa C, Barentsz JO. Head-to-head comparison of transrectal ultrasound-guided prostate biopsy versus multiparametric prostate resonance imaging with subsequent magnetic resonance-guided biopsy in biopsy-naïve men with elevated prostate-specific antigen: a large prospective multicenter clinical study. Eur Urol. 2019;75(4):570-8.

43. Monda SM, Vetter JM, Andriole GL, Fowler KJ, Shetty AS, Weese JR, Kim EH. Cognitive versus software fusion for MRI-targeted biopsy: experience before and after implementation of fusion. Urology. 2018;119:115-20.

\section{Publisher's Note}

Springer Nature remains neutral with regard to jurisdictional claims in published maps and institutional affiliations.
Ready to submit your research? Choose BMC and benefit from:

- fast, convenient online submission

- thorough peer review by experienced researchers in your field

- rapid publication on acceptance

- support for research data, including large and complex data types

- gold Open Access which fosters wider collaboration and increased citations

- maximum visibility for your research: over $100 \mathrm{M}$ website views per year

At BMC, research is always in progress.

Learn more biomedcentral.com/submissions 